\title{
18. Object Model for Planning and Scheduling Integration in Discrete Manufacturing Enterprises
}

\author{
Yasuyuki Nishioka ${ }^{1}$ \\ 1 Hosei University, Email: nishioka@k.hosei.ac.jp
}

\begin{abstract}
This paper proposes an object model for planning and scheduling integration in system development on discrete manufacturing. The model can deal with frequent changes of the market much more agile than the traditional models for production management. In addition, the object model can be translated to another model, which additionally has table, property and data type objects for automatic translation to a RDB schema. A modification procedure to adjust it to each case in industries is also described.
\end{abstract}

\section{INTRODUCTION}

Production management in manufacturing enterprises had been completely divided into two layers: an enterprise management layer in which order management and production/inventory planning are executed from a view of enterprise, and a shop floor management layer where production scheduling, quality control and maintenance activities are carried on in a decentralized basis. Facing to the current market environment, this division becomes an obstacle to achieve agile manufacturing that can synchronously respond to the market by means of dynamic change of operational decisions in shop floor management. Sales and services required to the enterprise should be responded to their customers eliminating the barrier between the two layers. Methods and models for such integrated systems are strongly required.

There were many investigations on this issue to integrate manufacturing systems as a whole enterprise model. For example, Purdue reference model for CIM was proposed for developing general model for manufacturing enterprises with respect to computer integrated systems (Williams, 1989). On the other hand, CIMOSA specification (ESPRIT, 1993) was proposed by an ESPRIT research project as a solution for enterprise integration supporting both model engineering and model execution. A framework of the Generalized Enterprise Reference Architecture (GERAM) proposed by the IFAC/IFIP task force was also a model considering the integration issue (IFIP-IFAC, 1999). As ontology, TOVE project proposed ontologies for enterprise modeling (Fox, 1998), which try to represent common vocabulary for modeling. In accordance with the Purdue model, ISA provided an industrial standard referred to as S95, which will also be an IEC/ISO international standard (ISA, 2000).

With respect to production planning and scheduling, all above approaches describe separate functionality of each module and very simple interfaces between them. Considering a production planning system and a detail scheduling system, 
information on the interface may have production orders sending to shop floors and their production results. However, those are too simplified in order to catch up the market changes. For example, because of a lack of data connection between production planning and detail scheduling, enterprises are due to have more inventory and production capacity than the necessary. Therefore, we are trying to make a new integration framework for production planning and scheduling (Nishioka, 2004).

Since traditional MRP/MRP II based planning systems have not been success to achieve such elaborate integration, advanced planning and scheduling (APS) architecture that deals with detail scheduling in a decision process of business layer is introduced. However, APS gets a limited success because it does not have a welldesigned integration model. A required integration model should deal with different granularities, different time spans and different focus points both on planning and scheduling.

This paper proposes an object model for planning and scheduling integration in manufacturing enterprises. The model is an extended version of the PSLX specifications published by PSLX consortium, which is a Japan based non profit organization for research and dissemination of general models and standards for next generation manufacturing industries (PSLX 2003). The PSLX specifications describe a decentralized architecture using a concept of autonomous agents, and define some ontologies used in their communication messages (Nishioka 2004).

The models in this paper are defined for discrete manufacturing where variety of parts and materials are composed into a final product in order to fulfill customer orders within shorter response time and less inventory. Many kinds of order processing such as make-to-order, configure-to-order, make-to-stock, and many kinds of production categories such as one-of-a-kind production, lot-production and repetitive production are in our scope.

This paper also discusses implementation issues on each manufacturer. To reduce the cost and the period of time for developing new/revised information systems, relationship between RDB schema and the proposed object model is discussed. A procedure to create an enterprise-specific RDB schema from a template of object models is also described. These procedural relationships are very important not only for the cost and time reduction but also for data federation or integration among different enterprises on a supply chain.

\section{ONTOLOGIES}

This section defines several important terms used in the object models. Since the terms have a variety of semantics depending on contexts of use, industrial categories, culture of shop floors and so on, the definitions are performing as ontology, which gives a meaning of itself providing a domain world without miss communications. Our approach to define ontology is to describe its intuitive explanations rather than precise specifications. However, they can be applied correctly to each particular situation, considering their structures and the relations to the expected decision processes.

\subsection{The primary terms}

The most primitive and important terms for planning and scheduling in a discrete manufacturing management are order, operation, item and resource. First, order is a 
term to represent an actual requirement of activities that are expected to do or already done on a certain period of time. There are a lot of order categories such as customer orders, prospective orders, purchase orders, production orders and work orders. Second, operation is a term to represent a class of specific activity for manufacturing in general. Activities for manufacturing generally include production operations, inspection operations, transportation operations and inventory operations.

Regarding that production operations usually produce and consume something goods or materials, item is a term to represent the something that is produced or consumed by an operation. Finally, resource is a term to represent an item that is necessary to carry out an operation but never consumed after its completion. Total capacity of a resource for each period of time can be shared by several operations that require the resource during their execution. Equipments, personnel and tools are typical classes of resources.

\subsection{Other useful terms for manufacturing}

There are several useful terms in addition to the primary terms. These are important for discussing production management not only from a local view of shop floor operations, but also from a view of enterprise-wide management. First, area is a term to represent an aggregation of resources, corresponding to a local decision making unit for manufacturing management. In addition, product is a term for a special item that is dealt with in business decisions. On the other hand, process is a term to represent an aggregation of operations that produce and/or consume products.

Special cases of resources can be defined as storage or route. Regarding that there are inventory operations and transportation operations, those operations require special classes of resources: storages and routes respectively. Storages are located at particular address and used to keep items for a particular period of time, while routes are connected to those storages and take account the traffic availability on each path. Finally, the term party is used to identify a border of an enterprise. Outside of the enterprise can be represented using customer and supplier that are sub classes of party.

\section{Terms for relational definitions}

Using the primitive terms described above, there are also definitions of several terms that represent relations between two of them. First, source is a term for a relation between product and area, representing possibility to supply the product. A relation between process and area is defined by a term loading, where the process needs to use capacity of the area during its execution period.

For operation, there are two terms for relative definitions. One is assignment, which represents a relation between operation and resource if the operation uses the resource. The other is material use, which represents a relation between operation and item that is consumed by the operation. In addition, there are other relations that define for two elements of a same kind. A term item structure presents a part-of relation between two items, if one item contains the other as a part or a material. On the other hand, precedence is a term that represents predecessor or successor of operations. 


\subsection{Terms for production management}

When a scheduler or planner is making a decision of production management, there are some special terms useful to understand the content of management. The followings are created every time to manage actual manufacturing processes. First of all, inventory and capacity should be addressed as very important terms. Inventory is a temporal status of storages or areas with respect to the volume of items or products respectively. Similar to this definition, capacity is a term for a temporal status of resources or areas to represent availability in making reservations for the current or future orders. Calendar is a special case of capacity, because it generally shows availability to work for each day.

In traditional production management, Master Production Schedule (MPS) and Material Requirement Plan (MRP) are very important terms. This paper also makes definitions for them as follows: MPS is a term to represent orders that actually request to produce some products within each period of time. On the other hand, $M R P$ is a term to represent actual requirements of materials that are needed for accomplishing production requests in MPS. Each requirement in the MRP corresponds to a purchase order, or other MPS if the material can be produced as a product in the same enterprise.

Finally, some additional technical terms for production management are defined using lot tracking and order pegging. Both of them are used to represent a relation between two orders. Lot tracking represents a connection from one order to another with respect to dependency of their actual production lot. This shows that a production lot produced by one work order is consumed by the other work order. Order pegging, on the other hand, represents a relation that is made for customer order fulfilment, connecting orders from a shop floor level to a business level.

\subsection{Definition of planning and scheduling}

Planning and scheduling is very general terms, thus we have to define their meanings to avoid any confusions. In order to do this, a concept of time, which is continuously going from the past to the future in the real world, is very important to distinguish them. With respect to time in this definition, scheduling can be defined as a term to represent a decision of time-dependent problems. The results of a decision contain parameters of time instances. On the other hand, planning is a term for decision making relate to activities in general. The definition of planning basically includes scheduling as a part. However, this paper discusses production planning as a time-independent problem, and considers that it occasionally communicates with scheduling for its time-dependent aspects.

Generally, production planning seems to have a time-dependent aspect. We think that most of all such time-dependent aspect can represent without continuous time horizon, using a concept of time bucket that represents a series of fixed-length periods of time. In a problem within a single time bucket, temporal parameters can be removed. A problem that lies across several time buckets can represent their time aspects using any other time-independent constraints.

Our definition requires that problems for some decision makings which contain time instances in its result should be solved by scheduling. In such cases, scheduling can be performed individually or as a portion of a planning problem. For example, a problem of order allocation to resources is categorized to a scheduling problem if it 
decides a particular start time and/or end time to reserve appropriate capacity of resources.

Since planning and scheduling have a lot of variety, the rest of this paper only focuses on production planning and production scheduling. In our model, production planning mainly deals with production order creation for many kinds of requests in manufacturers. Planning does not deal with continuous time horizon, but uses time buckets for some parameters if it needs temporal aspects. Production scheduling, then, mainly deals with work order assignment in a shop floor on a continuous time horizon.

\section{PLANNING AND SCHEDULING INTEGRATION}

This section briefly presents an integrated architecture of production planning and production scheduling. The architecture is based on the object model in which each object corresponds to the ontology defined in the previous section.

\subsection{Order management}

Before discussing the issue of integration, we have to clarify several groups of orders in manufacturing enterprises, because orders are very important information that performs in the integrated architecture. Especially, in a business layer, there are several types of orders, which are described as prospective orders, customer orders, purchase orders and production orders.

Prospective orders are created inside of an enterprise with respect to the future demand. Customer orders, on the other hand, are provided by customers, and will be connected to the prospective orders in cases where order-to-shipping lead time should be less than production lead time. For parts or materials that cannot be made in the enterprise, purchase orders are created in a procurement division. Finally, production orders are actual requirements of production activities that are aggregated for each production area. Production orders are created in a business layer, and also in a shop floor layer if necessary.

In a shop floor layer, there is another type of orders, which are referred to as work orders that deal with detail level of production due to the other orders such as production orders. A work order corresponds to particular activity that actually performs in a shop floor level. A work order can have time instances for its start time and end time. Therefore, scheduling deals with work orders that are producing a particular item in a shop floor. A work order can also represents any results of the activity.

As a special feature of our framework, production orders and purchase orders can be created and modified in shop floors. In other words, production planning and production scheduling communicate through these orders. This two-way information flow allows manufacturers to perform agility by means of collaboration of business divisions in an office and manufacturing divisions in distributed shop floors.

\subsection{Objects for planning and scheduling}

Regarding a manufacturing enterprise organization, we have been discussing two different layers: a business layer, which faces to customers of the enterprise, and a shop floor layer, which faces to reality of the shop floor controls. In terms of production management, functions of business divisions and functions of shop floors almost correspond to production planning and production scheduling respectively, 
because, business divisions are responsible to customer orders and make production orders for the customers, whereas shop floor managers try to fulfill the production orders by making a schedule.

Figure 1 illustrates our object model clarifying the border line of planning and scheduling. The outer area restricted by the dashed line represents a planning decision, while the area within the inner dashed line indicates objects related only to scheduling. Product, process and area are the planning objects as well as customer order and prospective order. The figure also shows that item, operation, resource and work order belong to scheduling objects. Production order and purchase order are on the border line, depending on the fact that they can be created by either planning or scheduling.

Relations between two of those objects in Figure 1 can also be pointed out. Some of them are defined in the previous section. For example, the relation between process and area represents loading. The relation between operation and resource represents assignment, and so on. In Figure 1, there are also self-referring relations such as precedence, item structure, order tracking and order pegging.

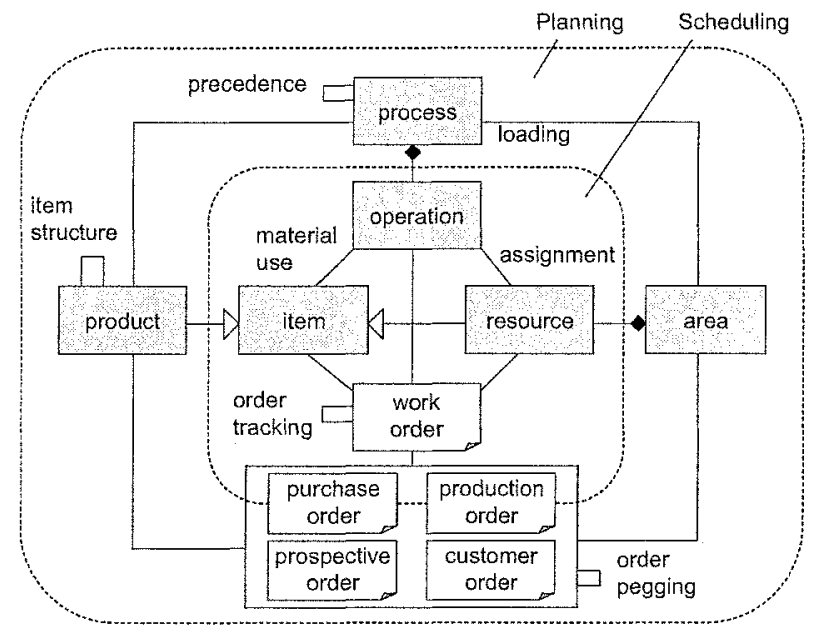

Figure 1: Primitive objects mapped on planning layer and scheduling layer

\subsection{Collaboration schema}

Using the objects in the model, our general framework of planning and scheduling integration is provided in Figure 2. This figure shows two modules of planning and scheduling and some relative objects to execute the modules as input or output. All types of orders indicated in Figure 1 can be shown in this chart. In addition to them, some transactional data such as capacity, inventory, pegging and tracking are addressed in the chart.

Collaboration between planning and scheduling is established through the feature that production orders and purchase orders can be made both by production planning and production scheduling. When a new production order is created in a shop floor, corresponding business processes will be carried out until the next cycle of production planning. In addition, inventory and capacity information are circulated 
as a bridge between planning and scheduling, while they have different granularities each of which corresponding to the different levels of planning and scheduling.

In conventional production management, production planning and detailed production scheduling perform on a sequential procedure. A production plan is decided first followed by a decision of scheduling that fulfills the production orders. Comparing to this, our model allows schedulers to make their own schedule even if it does not meet the given plan. They can create or modify production orders or purchase orders for themselves.

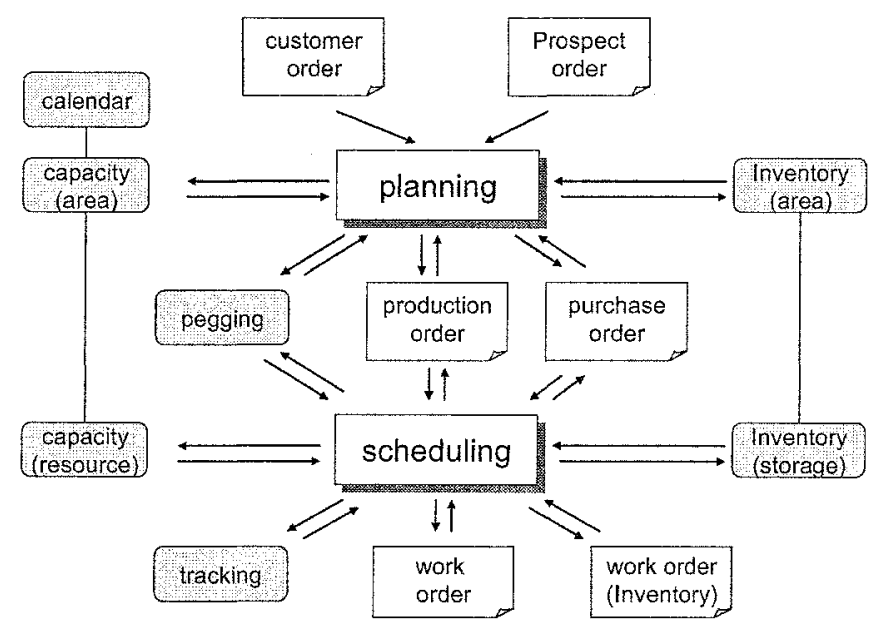

Figure 2: Integration framework of planning and scheduling

\subsection{Object hierarchy across the interface}

In order to establish two ways communication between production planning and production scheduling, there are object hierarchies across the interface. First of all, the hierarchies between item and product, resource and area, and operation and process are primarily important to make connections. All the scheduling level objects such as item, resource and operation should be represented as a child of the planning level objects. This is a kind of static relations across the interface.

In execution of production planning and production scheduling, order information is the key to coordinate the plan and the schedule. Consequently, work orders producing an item are defined in conjunction with a production order or customer order of a product. In this case, work order and production order refer to operation and process respectively. This dynamic relationship is represented by pegging objects that are generated and maintained by the planning and scheduling modules collaboratively.

The final and most meaningful relations are the self referring hierarchy in capacity objects and inventory objects. There are at least two levels in capacity objects: an area level capacity and a resource level capacity. Usually area capacity corresponds to capacity of a bottleneck resource in the area. On the other hand, inventory also has two levels: area level inventory and storage inventory. Area level inventory represents the volume of particular products or materials regardless of their status whither they have been made or not. In other wards, the number of 
inventory increases when the substance comes inside the area and decreases when it goes out from the area. One layer's information of inventory or capacity is effective as a constraint of the other, and vice versa.

\section{IMPLEMENTATION ISSUES}

In order to apply the model to real industries, system development usually needs a RDB schema, which can be also represented by object models. However, the detail of the schema is different from the general model because it should have technology specific aspects such as efficiency of table search. This section describes how to translate the general model to appropriate objects of RDB schema.

\subsection{Object models and RDB}

Considering RDB schema, other objects can be represented with respect to table information, property information and data type information. Considering RDB implementation, we define four object classes: master table, relation table, order table and other data table. All tables in RDB used for planning and scheduling will be implemented as a table of those four, having information defined in one of the general objects described in the previous sections.

Regarding RDB features, all master tables have property information about id, description, price, cost, capacity and registration. For the primary objects, we define sub classes of the master including party master, area master, storage master, item master, process master, operation master and resource master. Relation tables that relate one object to another are defined including source table, assignment table, loading table, material use table, item structure table, and precedence table.

Table for orders can be defined using prospective order table, customer order table, purchase order table, production order table, and work order table. This classification is the same as the object model. Note that these order tables can represent not only orders but also the results or progress of the orders.

Finally, the transactional data generated during the planning and scheduling decisions are created as the other data classes. They include MPS table, MRP table, lot tracking table, order pegging table, inventory table, capacity table, and calendar table. The lot tracking table and the order pegging table have links to two orders, connecting one to the other. The inventory table and the capacity table perform in both a planning and a scheduling.

\subsection{Table generation procedure}

The object model modified for RDB implementation does not always meet particular cases in the real manufacturing. Therefore, we provided a typical model as a template, which can vary depending upon each environment. In order to adjust the model, we propose the following procedure.

\section{Step 1: Create and delete primitive objects for the application}

Many kinds of objects may be added to the template for practical industrial applications. Some of them come from a schema in legacy systems. Furthermore, it is necessary to delete some template objects that are not used in the application. Sometimes, separation of an existing object is required. Sometimes, two objects are merged. 


\section{Step 2: Modify relational information among the objects}

Relations among primitive objects can be changed. Depending on the cardinality of relationship between two objects, some relational objects are deleted where the target number can be reduced to single. Some new relational objects are created where one-to-many relations are additionally required.

\section{Step 3: Modify properties for each object}

In order to modify detail information of a table object, you can attach and/or detach any property candidates to the table object. New property candidates can be created if necessary. The content of each property of tables can be modified by choosing its attributes.

\section{Step 4: Move or duplicate attribute through the links}

With respect to efficiency of the RDB, some attributes of properties need to move or duplicate from one table object to other through the directed link between them. For example, consider that an item table has a link to a storage table, and then the storage table has a property of quantity. In this case, the property of quantity can move to the item table, so that the item table directory shows the inventory amount.

\section{Step 5: Define data type object for each attribute}

The attributes of each property have a particular data type such as string, integer, float, Boolean, and so on. The final specification of the data type completely depends on RDB management systems. Since the data type in object model represents in a conceptual level, the final data types of RDB systems should be determined.

\section{CONCLUDING REMARKS}

As industrial case studies, we investigated three application systems for different kinds of manufacturers: a 2nd tier automotive manufacture, which repetitively makes pipes for gasoline, oil and air supply; a manufacture, which provides one-ofa-kind laser cutting fabrication services for high precision parts; and a pump manufacturing enterprise, which provides their products in make-to-order basis. After the investigation of the detail of the current RDBs, the whole part of the data in the three enterprises can be represented by our object model with some appropriate extensions. Their object models also regenerated the corresponding $\mathrm{RDB}$ schema according to the procedure.

This experimental study showed that the proposed object model is practical enough for real industries. Furthermore, we found out that representations using the model are much easy to understand while we discuss the additional modules for the enterprises. Unfortunately, those three enterprises did not have a scheduling module as a computer system. They made daily schedules on white boards or on paper charts. However, in this case study, we successfully made preliminary design of database for those scheduling systems according to the object model.

From the experiment, this paper argues that the approach using the object model for planning and scheduling integration is more successful than the past. In traditional approach, in which a planning system and a scheduling system are developed independently, interfaces are so simple that any special advantages can not be provided from the integration. Moreover, in terms of step-by-step development, the 
representation of the object model is more useful because of independence between the computer-specific implementation aspects and the object model in manufacturing enterprises. The three enterprises of the case studies keep improving the system development according to this framework.

This paper proposed an object model for planning and scheduling integration in practical fields of discrete manufacturing. Different from the traditional system of production management, the integrated model was defined in order to adjust the plan and schedule collaboratively to the frequent changes of the market. With respect to system development for real industries, the object model can be translated to another object model for RDB schema, which has special features of tables, properties and data types. Since the RDB dependent model was provided as a template for any modification according to each case of manufacturing enterprises, we also proposed a modification procedure to meet each requirement in the industries.

\section{Acknowledgments}

This study is partially depending on the valuable discussions in the project of PSLX consortium. Author appreciates all the contribution of PSLX technical committees and the other members of the project.

\section{REFERENCES}

ESPRIT Consortium AMICE (Eds.) (1993), CIMOSA: Open System Architecture for CIM, Springer-Verlag

Fox, M.S., Gruninger, M., (1998), Enterprise Modelling, AI Magazine, AAAI Press, Fall 1998, pp. 109-121

IFIP-IFAC Task Force on Architectures for Enterprise Integration (1999), GERAM: Generalised Enterprise Reference Architecture and Methodology, [http://www.cit.gu.edu.au/-bernus/taskforce/geram/versions/geram1-63/v1.6.3.html]

ISA (2000), Enterprise-Control System Integration Part 1: Models and Terminology, ISA-95.00.01, The Instrumentation, Systems, and Automation Society

Nishioka, Y. (2004), Collaborative agents for production planning and scheduling (CAPPS): a charange to develop a new software system architecture for manufacturing management in Japan, International Journal of Production Research, (to appear).

PSLX consortium (Ed.) (2003), PSLX Engineering specification, PSLX consortium, [http://www.pslx.org]

Williams, T. J. (Eds.) (1989), A reference model for computer integrated manufacturing (CIM), Instrument Society of America 